\title{
High temperature resistivity characteristics of nonstoichiometric $\mathrm{V}_{2} \mathrm{O}_{3}{ }^{*}$
}

\author{
H JHANS and $J$ M HONIG \\ Department of Chemistry, Purdue University, West Lafayette, Indiana 47907, USA \\ MS received 16 September 1981
}

\begin{abstract}
The resistivity anomaly encountered in stoichiometric $\mathrm{V}_{2} \mathrm{O}_{3}$ is gradually suppressed with increasing density of cation vacancies. These observations are discussed in terms of related findings reported in the literature.
\end{abstract}

Keywords. Stoichiometric $\mathrm{V}_{2} \mathrm{O}_{3}$; resistivity; cation vacancies.

\section{Introduction}

It is by now well established that incorporation of excess oxygen into $\mathrm{V}_{2} \mathrm{O}_{3}$ generates cation vacancies, and that this process leads to serious disruptions in structure (Nakahira et al 1970; Novikov et al 1971; McWhan et al 1971; Nagasawa et al 1972 ; Ooshima 1973 ; Shirasaki and Ooshima 1973 ; Kuwamoto and Honig 1980 ; Ueda et al 1980), transport phenomena (Novikov et al 1971; McWhan et al 1971 ; Kozyreva et al 1972 ; Nagasawa et al 1972 ; Kimizuka et al 1973 ; Noguchi et al 1973 ; Launay et al 1976 ; Kuwamoto and Honig 1980 ; Ueda et al 1980), magnetic properties (Nakahira et al 1970; McWhan et al 1971; Zubkov et al 1972; Ooshima 1973; Ueda 'et al 1977,1978,1980), and optical characteristics (Novikov et al 1971 ; Kozyreva et al 1972). Prior work has concentrated on variations of electrical and magnetic characteristics and on changes in the metal-antiferromagnetic insulator transition of $\mathrm{V}_{2(1-y)} \mathrm{O}_{3}$ at low temperatures $T<300 \mathrm{~K}$ for $0 \leq y<0.01$.

We report here briefly on alterations in electric properties in the range $300-900 \mathrm{~K}$ which arise from altering the oxygen/vanadium ratio in $\mathrm{V}_{2} \mathrm{O}_{3}$; this encompasses the region $400-600 \mathrm{~K}$ where stoichiometric $\mathrm{V}_{2} \mathrm{O}_{3}$ exhibits an anomalous rise in resistivity with temperature, first noted by Feinleib and Paul (1967), and subsequently verified by other investigators (Novikov et al 1971; McWhan et al 1971 ; Kozyreva et al 1972; Pettifer et al 1973; Honig et al 1974; Chandrashekar et al 1975; Kuwamoto et al 1980). These results will then be briefly discussed in the wider context of charge transport and metal-insulator transition mechanisms in $\mathrm{V}_{2} \mathrm{O}_{3}$.

"Research supported by NSF-MRL Grant DMR 77-23798. 


\section{Experimental}

Single crystal specimens of appropriate size were cut from $\mathrm{V}_{2} \mathrm{O}_{3}$ boules that had been grown in a skull melter and were annealed in a variety of $\mathrm{CO} / \mathrm{CO}_{2}$ atmospheres to achieve different stoichiometries. The details of this procedure are discussed at length in preceding publications (Katsura and Hasegawa 1967; Wakihara and Katsura 1970 ; Shivashankar et al 1981).

Four probe resistivity measurements were then carried out in an automatic set up which provided for periodic reversal of the current direction so as to permit averaging out of spurious thermoelectric voltages. Silver paint was used for the attachment of leads. The results for three specimens are displayed in figure 1 as plots of resistivity $\rho v s$. temperature $T$. Also included is an earlier set of measurements (Chandrashekhar et al 1975; Kuwamoto et al 1980) on $\mathrm{V}_{2} \mathrm{O}_{3}$ specimens grown in an arc melter (Fan and Reed 1972).

It is seen that the earlier results are in good accord with present measurements carried out on the most nearly stoichiometric material. Furthermore, with increasing departure from strict stoichiometry the size of the electric anomaly is reduced, and ultimately the anomaly is eliminated.

\section{Discussion}

The trend noted in figure 1 is similar to that encountered in the $\left(\mathrm{V}_{1-x} \mathrm{~T} \mathrm{~T}_{x}\right)_{2} \mathrm{O}_{3}$ system with increasing Ti content (Chandrashekhar et al 1975). Here, too, the

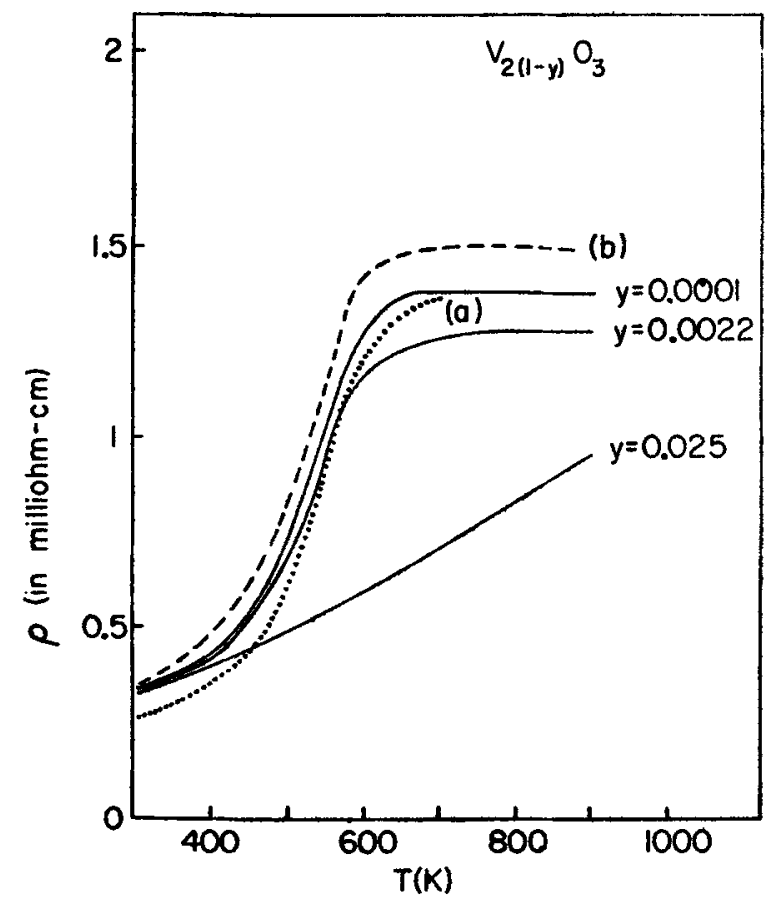

Figure 1. Resistivity $(\rho)$ versus temperature $(T)$ for $\mathrm{V}_{2}(1-y) \mathrm{O}_{3}$ single crystals $(0 \leq y \leq 0.025)$. The results for as-grown single crystals of $\mathrm{V}_{2} \mathrm{O}_{3}$ are shown for comparison. Curve (a) (after Chandrashekhar et al 1975); Curve (b) (after Kuwamoto et al 1980). 


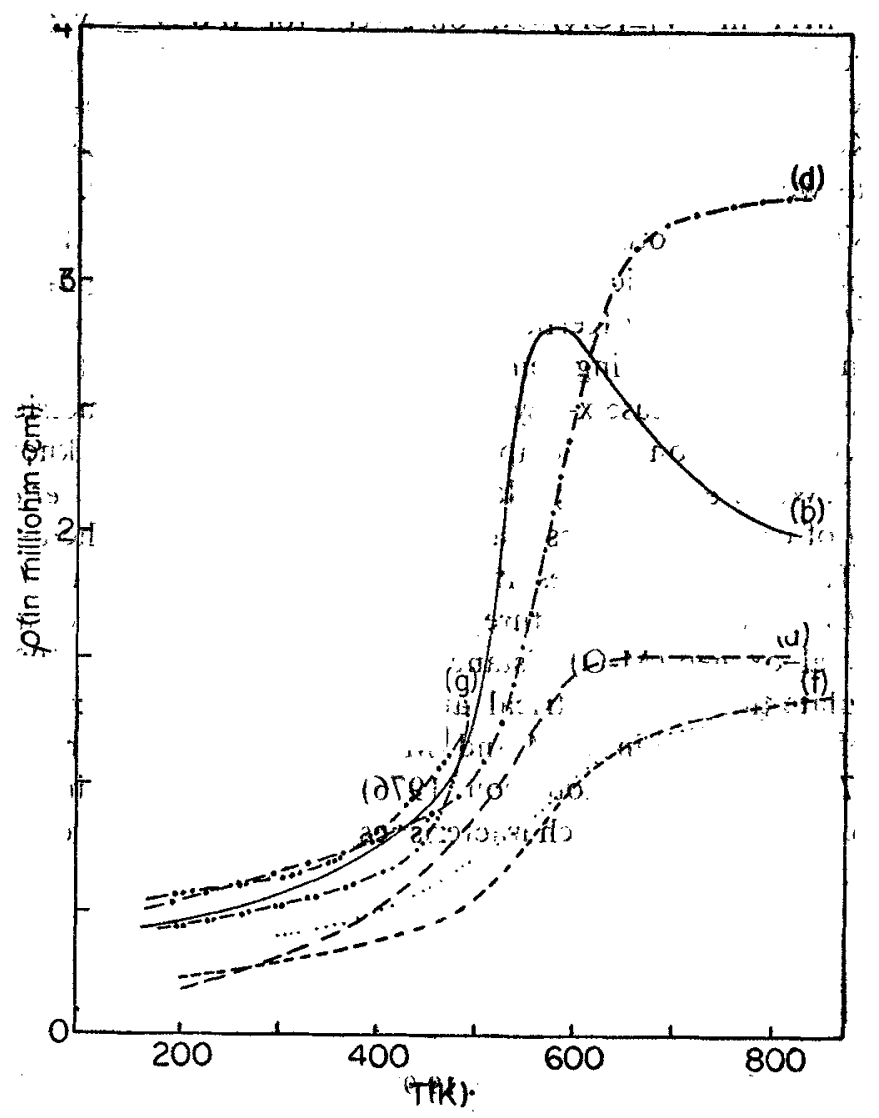

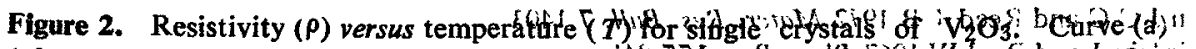

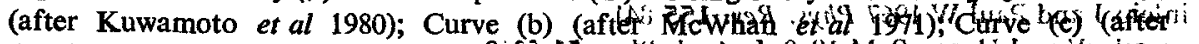

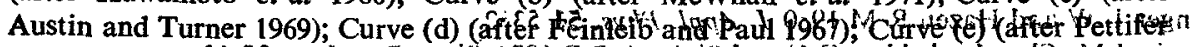

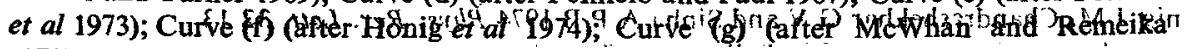
1970).

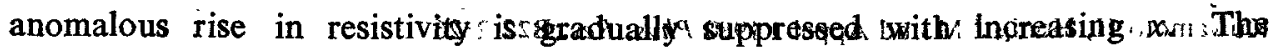
similarity in behaviour perhaps

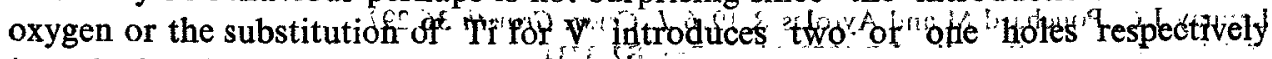
into the band structure of the $Y_{2} P_{3}$ host:

The present data also merit roompatison with cearlier bwork lassembleding figure 24

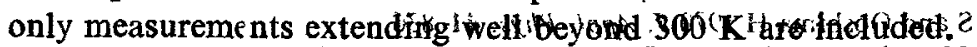

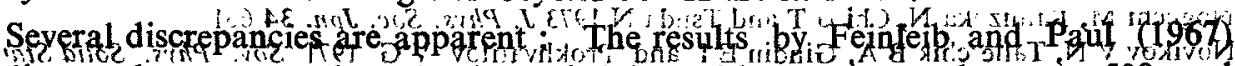
indicate a larger anomalous rise in resistivity with temperature between $50 \theta_{s}$ and $700 \mathrm{~K}$ than was observed by other workers. Ther beasuramenta

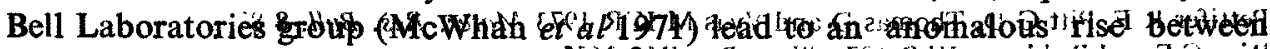

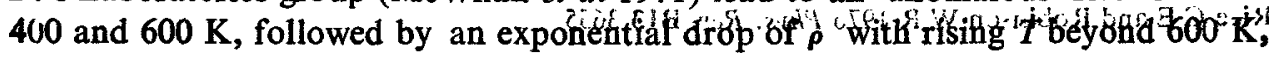


This latter feature has been interpreted by the authors as indicating that a continuous metal-insulator transition (MIT) takes place; this represents the extension of the sharp MIT in $\left(\mathrm{V}_{1-x} \mathrm{Cr}_{x}\right)_{2} \mathrm{O}_{3}$, observed for $0.005 \leq x \leq 0.0018$, to the supercritical region. Such an interpretation is buttressed by nuclear magnetic relaxation measurements on $\mathrm{V}_{2} \mathrm{O}_{3}$ (Kerlin et al 1973). These were found to satisfy the Korringa relation below $550 \mathrm{~K}$ and to become independent of $T>550 \mathrm{~K}$, as would be expected for a paramagnetic insulator.

However, since many other investigators have not observed features in the electrical properties that indicate the occurrence of a MIT in $\mathrm{V}_{2} \mathrm{O}_{3}$, the claims by McWhan et al (1971) and by Kerlin et al (1973) should be accepted with caution. The electric anomaly with rising temperature cannot be directly correlated with variations in structure because $\mathrm{x}$-ray diffraction on nonstoichiometric $\mathrm{V}_{2} \mathrm{O}_{3}$ has not been studied as a function of temperature. However, it is known (Nakahira ct al 1973; McWhan et al 1971 ; Nagasawa et al 1972; Ueda et al 1980) that the generation of cation vacancies leads to a contraction of the $c$ - and $a$ - lattice parameters. This is also the result of increasing the pressure (McWhan and Remeika 1970) at rosm temperature, leading to a lessening of all metal-metal (M-M) and me:al-oxygen (M-O) distances (Finger and Hazen 1980). One can therefore speculate that the electrical anomaly in stoichiometric $\mathrm{V}_{2} \mathrm{O}_{3}$, being a concomitant of an increase in $\mathrm{M}-\mathrm{M}$ and $\mathrm{M}-\mathrm{O}$ distances with rising temperature (Robinson 1975 ; Rice and Robinson 1976), is suppressed in cation-deficient material because its structural characteristics approach those of metallic $\mathrm{V}_{2} \mathrm{O}_{3}$ under hydrostatic compression.

\section{References}

Austin I G and Turner C E 1969 Philos. Mag. 19939

Chandrashekhar G V, Shin S H, Jayaraman A, Keem J E and Honig J M 1975 Phys. Status Solidi A29 323

Fan J C C and Reed T B 1972 Mater. Res. Bull. 71403

Feinleib J and Paul W 1967 Phys. Rev. 155841

Finger L W and Hazen R M 1980 J. Appl. Phys. 515362

Honig J M, Chandrashekhar G V and Sinha A P B 1974 Phys. Rev. Lett. 3213

Katsura T and Hasegawa M 1967 Bull. Chem. Soc. Jpn. 40561

Kerlin A L, Nagasawa H and Jerome D 1973 Solid State Commun. 131125

Kimizuka N, Ishii M, Saeki M, Nakano M and Nakahira M 1973 Solid State Commun. 1243

Kozyreva M S, Novikov V N and Tallerchik B A 1972 Sov. Phys. Solid State 14639

Kuwamoto H, Honig J M and Appel J 1980 Phys. Rev. B22 2626

Kuwamoto $H$ and Honig J M $1980 \mathrm{~J}$. Solid State Chem, 32335

Launay J C, Pouchard M and Ayroles R 1976 J. Cryst. Growth 36297

McWhan D B and Remeika J P 1970 Phys. Rev. B2 3734

McWhan D B, Menth A and Remeika J P 1971 J. Phys. (Paris) Colloq. 32 C1-1079

Nagasawa K, Bando Y and Takada T 1972 J. Cryst. Growth 17143

Nakahira M, Horiuchi S and Ooshima H 1970 J. Appl. Phys. 41836

Noguchi M, Kimizuka N, Chiba T and Tsuda N 1973 J. Phys. Soc. Jpn. 34661

Novikov V N, Tallerchik B A, Gindin E I and Prokhvatilov V G 1971 Sov. Phys. Solid State 122061

Ooshima H 1973 Yogyo Kyoka Shi 81162

Pettifer R F, Pitt G D, Thomas D and Vyas M K. R 1973 Mater. Res. Bull. 887

Rice C E and Robinson W R 1976 Phys. Rev. B13 3655 
Robinson W R 1975 Acta Crystallogr. B31 1153

Shirasaki S and Ooshima H 1973 Muki Zaishitsu Kenkyusho Kenkyu Hokokusho 1152

Shivashankar S A, Aragon R, Harrison H R, Sandberg C J and Honig J M 1981 J. Electrochem. Soc, 1282472

Ueda Y, Kosuge K, Kachi S, Shinjo T and Takada T 1977 Mater. Res. Bull. 1287

Ueda Y, Kosuge K, Kachi S, Yasioka H, Nishihara H and Heidemann H 1978 J. Phys. Chem. Solids 391281

Ueda Y, Kosuge K and Kachi S $1980 \mathrm{~J}$. Solid State Chem. 31171

Wakihara $M$ and Katsura T 1970 Metall. Trans. 1363

Zubkov V G, Matveenko I I, Tallerchik B A and Gel'd P V 1972 Sov. Phys. Solid State 132885 